\title{
Synthesis of Mo, W, and Mo- and W-Doped Multiwall VONTs via Sol-Gel and Hydrothermal Methods
}

\author{
Roya Rouhani, ${ }^{1}$ Hamid Reza Aghabozorg, ${ }^{2}$ \\ Mohsen Asadi Asadabad, ${ }^{3}$ and Hossein Aghabozorg ${ }^{4}$ \\ ${ }^{1}$ Young Researchers Club, North Tehran Branch, Islamic Azad University, Tehran, Iran \\ ${ }^{2}$ Research Institute of Petroleum Industry, NIOC, Tehran, Iran \\ ${ }^{3}$ Material Research School, NSTRI, Isfahan, Iran \\ ${ }^{4}$ Chemistry Department, Teacher Training University, Tehran, Iran
}

Correspondence should be addressed to Roya Rouhani; r.rouhani@yahoo.com

Received 7 June 2012; Revised 27 August 2012; Accepted 10 September 2012

Academic Editor: Beatriz Royo

Copyright (C) 2013 Roya Rouhani et al. This is an open access article distributed under the Creative Commons Attribution License, which permits unrestricted use, distribution, and reproduction in any medium, provided the original work is properly cited.

\begin{abstract}
Mo, W, and Mo and W were doped into multiwall vanadium oxide nanotubes. The syntheses were performed using sol-gel method followed by hydrothermal method. The synthesized samples were characterized by XRD, SEM, EDX, and TEM techniques. The XRD patterns of the synthesized samples indicated that Mo and W could be doped into VONTs totally up to 50\%. The SEM and TEM images showed that the prepared samples have tubular and multiwall morphology and open ends.
\end{abstract}

\section{Introduction}

Inorganic nanotubes have potential application in catalytic processes and electrochemical devices [1,2]. In recent years, inorganic nanotubes have received significant attention $[3,4]$. Vanadium oxide nanotubes (VONTs) are one kind of inorganic nanotubes that have application in batteries, sensors, catalysts, and electrochemical devices [5-7]. These nanotubes have been synthesized with various methods such as sol-gel, and hydrothermal $[8,9]$. Doping of transition metals into these nanotubes can improve their properties for the desired application for example, "Mo-doped vanadium oxides have found a wide range of applications because of their selective oxidation as well as the unique interaction between $\mathrm{V}_{2} \mathrm{O}_{5}$ and $\mathrm{MoO}_{3}$ owing to the similarity of ionic radii and the structures in their highest oxidation state" [9]. Recently, the properties of mixed vanadium-tungsten oxides have been investigated due to their potential use in electrochromic devices $[8,10]$. Thus, by doping of both Mo and W into VONTs, new properties for VONTs could be achieved. In this work, Mo doped VONTs up to $20 \mathrm{~mol} \%$ and $\mathrm{W}$ doped VONTs up to $20 \mathrm{~mol} \%$ and for the first time Mo and W simultaneously were doped into VONTs up to $50 \mathrm{~mol} \%$.

\section{Material and Method}

$\mathrm{V}_{2} \mathrm{O}_{5}$ ( $>99 \%$, Merck), $\mathrm{MoO}_{3}$ (<99\%, Merck), $\mathrm{H}_{2} \mathrm{WO}_{4}$ ( $<98 \%$, Merck), and $\mathrm{C}_{18} \mathrm{H}_{39} \mathrm{~N}$ ( $\sim 90 \%$, Merck) as a template were used for syntheses of the desired materials. For preparation of $\left(\mathrm{V}_{1-x} \mathrm{M}_{x}\right)_{y}$ ONTs $(\mathrm{M}=\mathrm{Mo}, \mathrm{W}$, and $\mathrm{Mo}$ and $\mathrm{W})$ compounds stoichiometric amount of the desired reactants were mixed in distilled water, and the mixture were stirred for $48 \mathrm{~h}$ in air. The resulting mixture (gel) was transferred into a teflon-lined autoclave with stainless steel shell. The autoclave was kept at $185^{\circ} \mathrm{C}$ for 7 days and then allowed to cool naturally. The obtained product was washed with distilled water and absolute ethanol and then dried at $80^{\circ} \mathrm{C}$ for $8 \mathrm{~h}$. X-ray powder diffraction (XRD) patterns of the prepared samples were obtained using STADI MP X-ray diffractometer with $\mathrm{Cu} \mathrm{K} \alpha$ radiation $(\lambda=$ $1.5406 \AA$ ). The morphology and quantitative analysis of the samples were studied by scanning electron microscopy (SEM) on Philips XL30 microscope equipped with energydispersive X-ray spectroscopy (EDX) and transmission electron microscopy (TEM) on Philips EM208S microscope operated at $100 \mathrm{kV}$. 


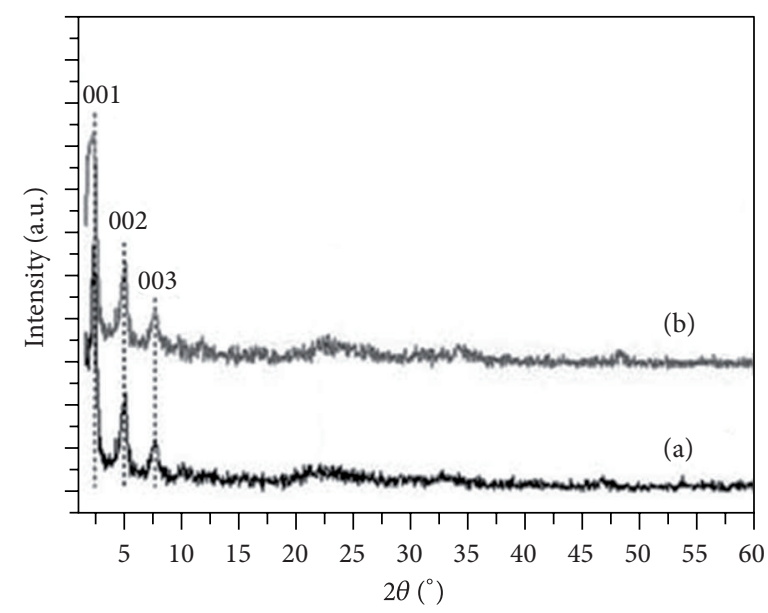

FIGURE 1: XRD patterns of VONTs (a) and Mo-doped VONTs [9] (b).

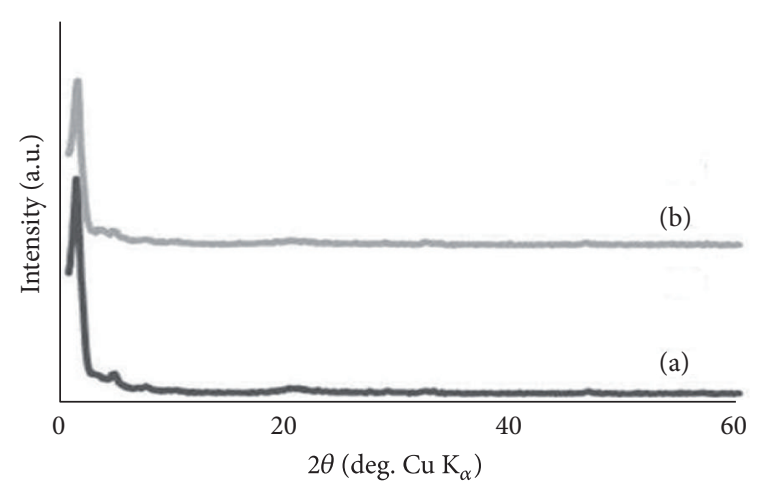

(A)

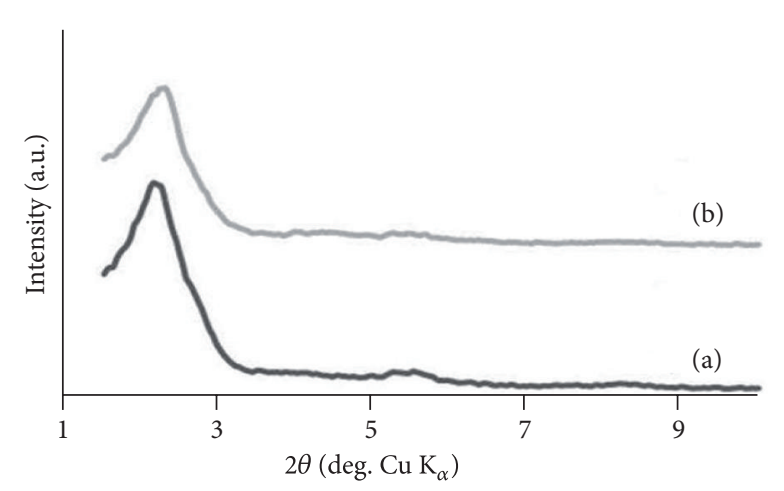

(B)

FIgURE 2: XRD patterns of (a) $\mathrm{V}_{0.9} \mathrm{Mo}_{0.1}$ ONTs and (b) $\mathrm{V}_{0.8} \mathrm{Mo}_{0.2}$ ONTs at two different ranges of $2 \theta$ ((A) $2 \theta=1-60^{\circ}$ and (B) 2 $\left.\theta=1-10^{\circ}\right)$.

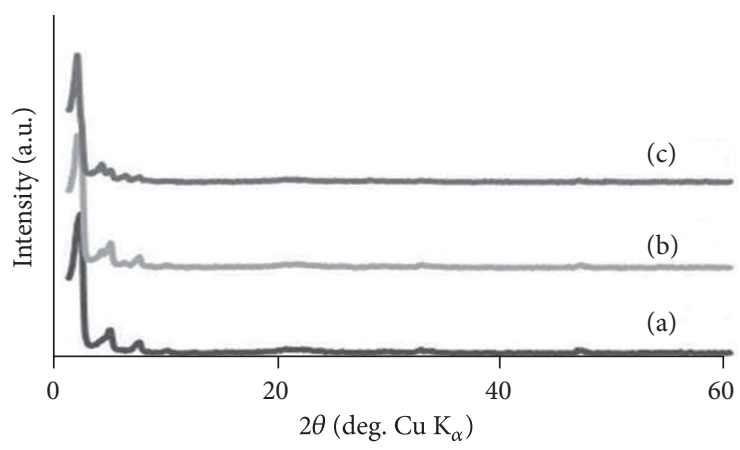

(A)

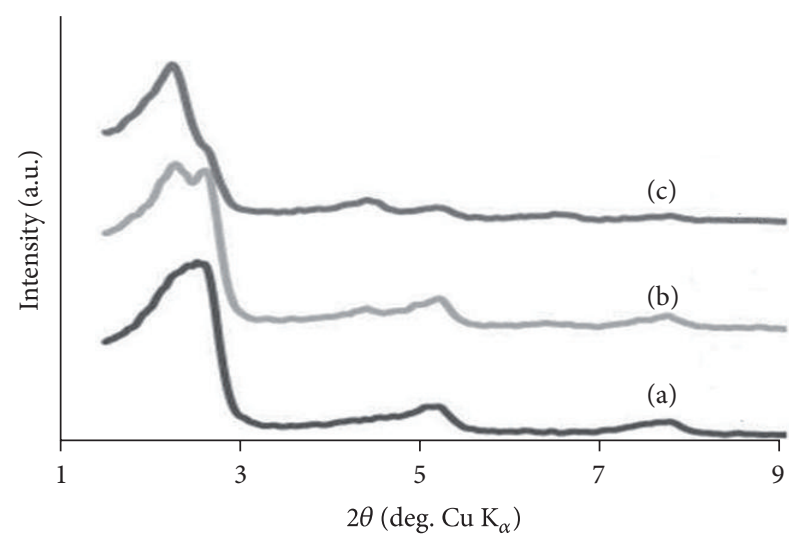

(B)

FIGURE 3: XRD patterns of (a) $\mathrm{V}_{0.95} \mathrm{~W}_{0.05}$ ONTs, (b) $\mathrm{V}_{0.9} \mathrm{~W}_{0.1}$ ONTs, and (c) $\mathrm{V}_{0.8} \mathrm{~W}_{0.2}$ ONTs at two different ranges of $2 \theta\left((\mathrm{A}) 2 \theta=1-60^{\circ}\right.$ and (B) $2 \theta=1-10^{\circ}$ ).

\section{Results}

The X-ray diffraction (XRD) patterns of $\mathrm{V}_{1-x} \mathrm{Mo}_{x} \mathrm{ONTs}(x=$ 0.1 and 0.2$), \mathrm{V}_{1-x} \mathrm{~W}_{x} \mathrm{ONTs}(x=0.05,0.1$ and 0.2$)$, and $\mathrm{V}_{1-(x+y)} \mathrm{Mo}_{x} \mathrm{~W}_{y}$ ONTs $(x+y=0.1,0.2,0.4$ and 0.5$)$ are shown in Figures 2, 3, and 4, respectively. The peaks at $2 \theta>$ $10^{\circ}$ originate from the two dimensional structure of the walls and nanotubes layers. The XRD patterns of the prepared samples and the comparison of these patterns with that of VONTs $[11,12]$ (Figure 1) indicate that desired species are 


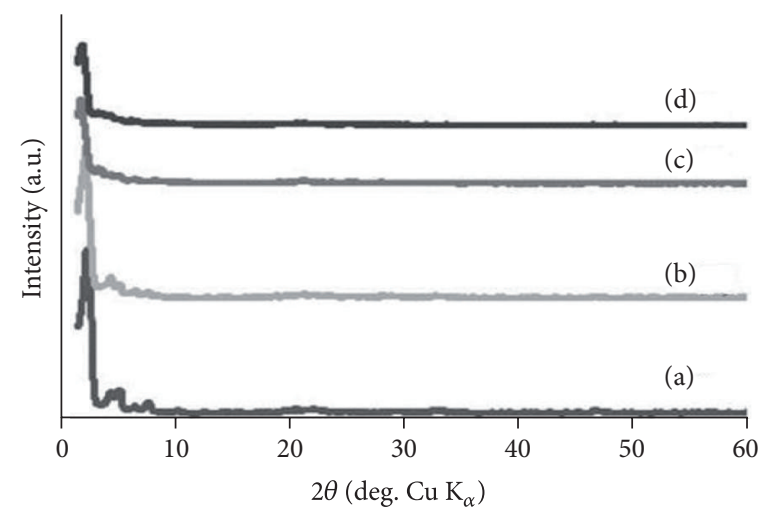

(A)

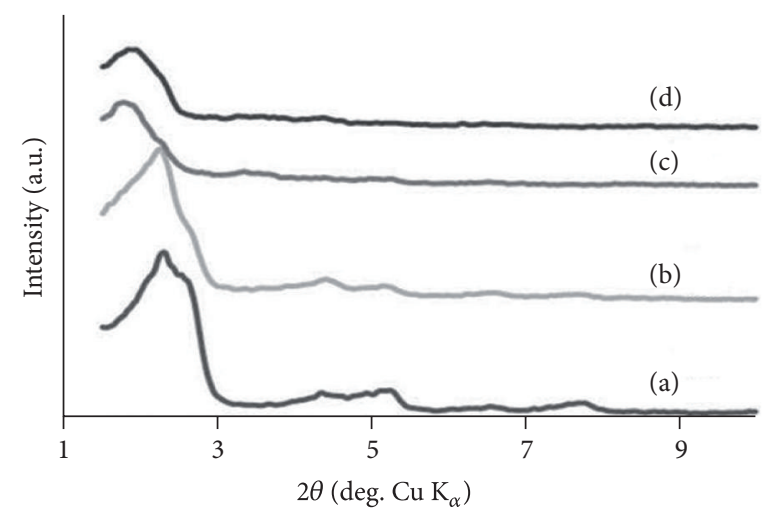

(B)

FIGURE 4: XRD patterns of (a) $\mathrm{V}_{0.9} \mathrm{Mo}_{0.05} \mathrm{~W}_{0.05}$ ONTs, (b) $\mathrm{V}_{0.8} \mathrm{Mo}_{0.1} \mathrm{~W}_{0.1}$ ONTs, (c) $\mathrm{V}_{0.6} \mathrm{Mo}_{0.2} \mathrm{~W}_{0.2} \mathrm{ONTs}$, and (d) $\mathrm{V}_{0.5} \mathrm{Mo}_{0.25} \mathrm{~W}_{0.25}$ ONTs at two different ranges of $2 \theta\left((\mathrm{A}) 2 \theta=1-60^{\circ}\right.$ and (B) $\left.2 \theta=1-10^{\circ}\right)$.

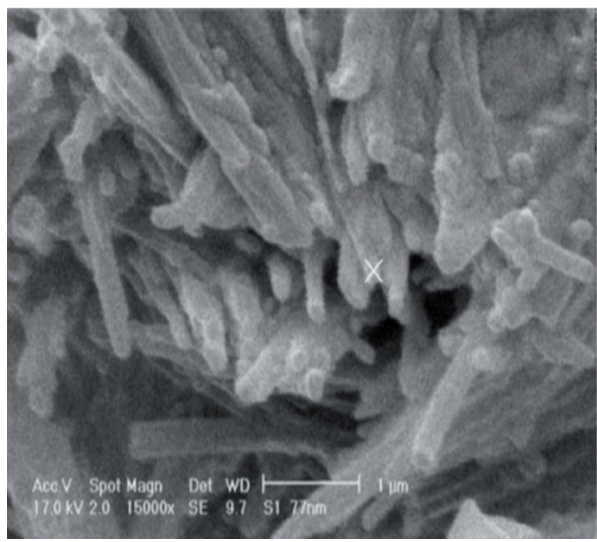

(a)

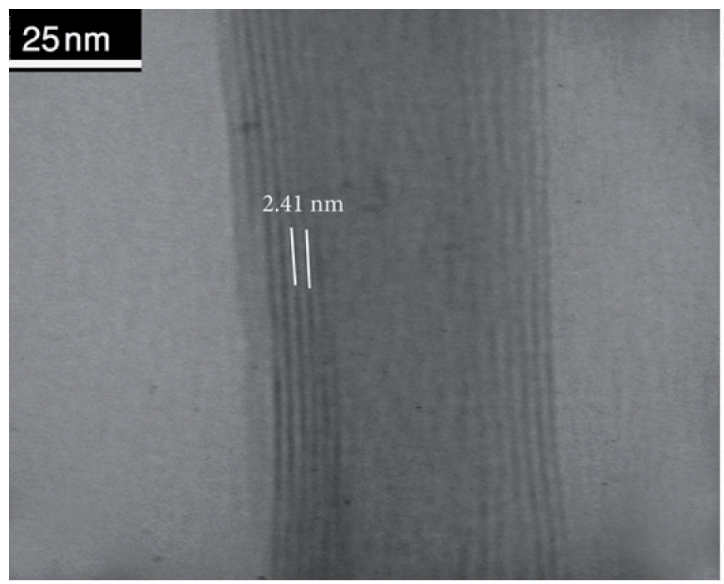

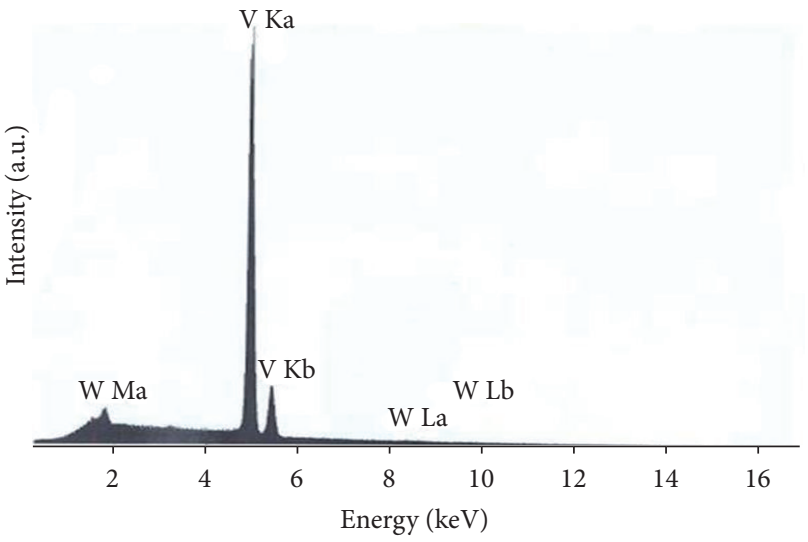

(b)

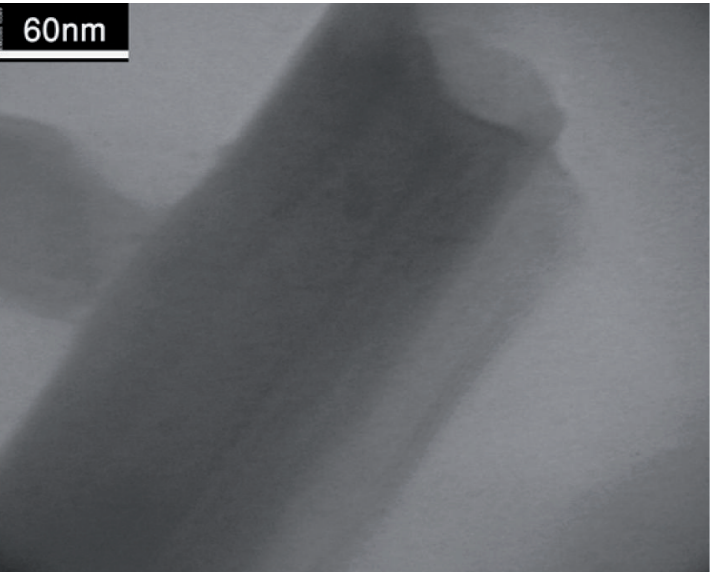

(c)

Figure 5: (a) SEM image, (b) EDX spectrum, and (c) TEM images of $\mathrm{V}_{0.95} \mathrm{~W}_{0.05} \mathrm{ONTs}$.

doped into the vanadium oxide nanotubes. The peak with the highest intensity at the low diffraction angle $\left(2 \theta<10^{\circ}\right)$ reflects the inter layer distances of the nanotubes [13]. These patterns indicate that the increase of doping level of the elements into VONTs leads to increasing interlayer distances (Figures 2, 3, and 4) which are considered to be due to the replacement of $\mathrm{V}$ in vanadium oxide nanotubes by Mo and $\mathrm{W}$ with larger ionic radii.

Table 1 shows the $d_{\mathrm{XRD}}$ values of the most intensive peak for different samples.

The $d$ value of $\mathrm{V}_{0.9} \mathrm{~W}_{0.1} \mathrm{ONTs}$, is relatively similar to that of $\mathrm{V}_{0.9} \mathrm{Mo}_{0.05} \mathrm{~W}_{0.05}$ ONTs and the $d$ value of $\mathrm{V}_{0.8} \mathrm{~W}_{0.2}$ ONTs is 


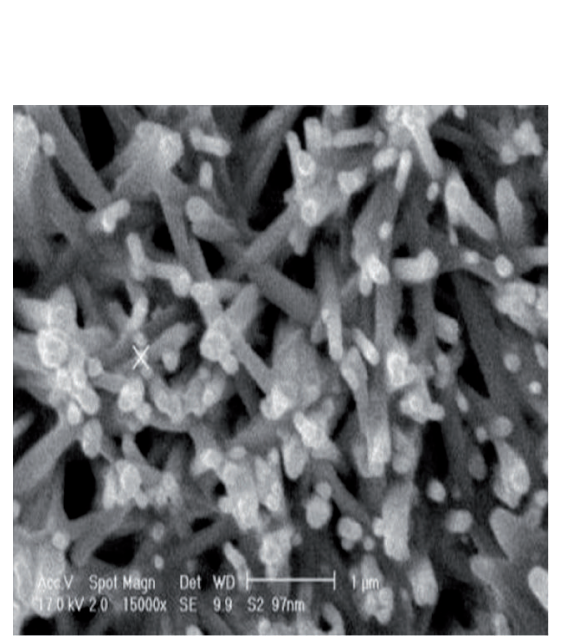

(a)

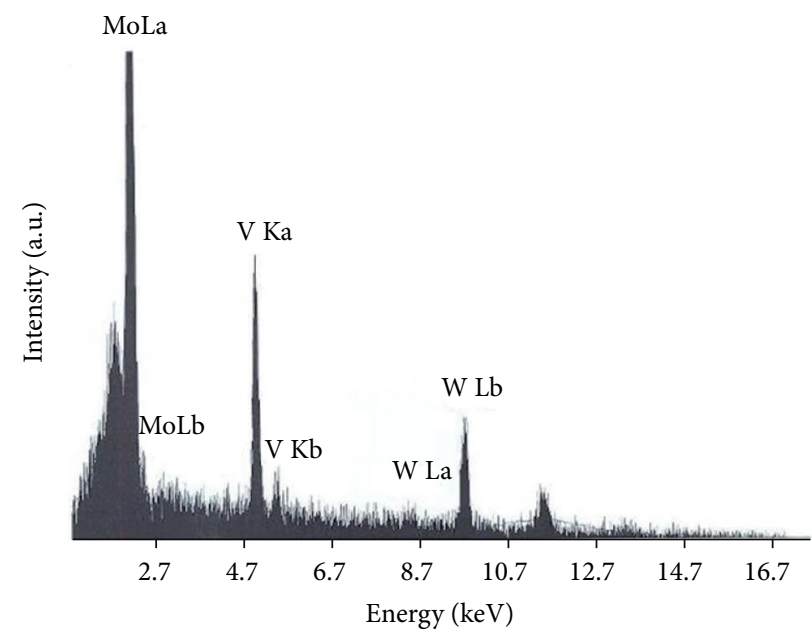

(b)

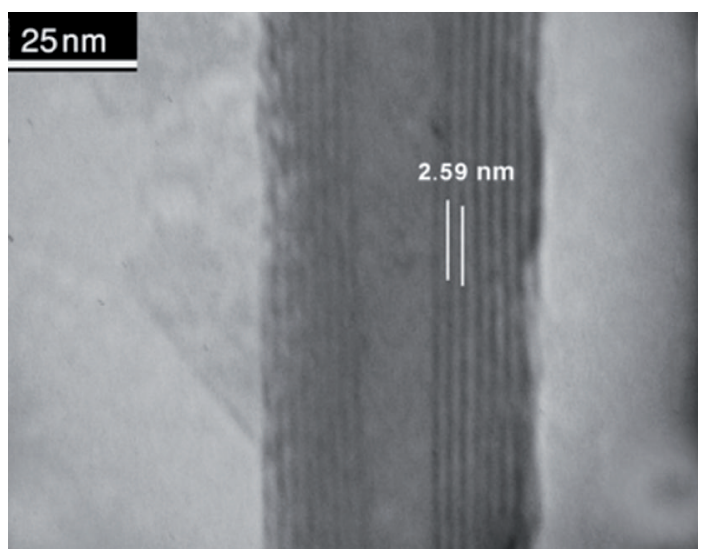

(c)

FIgure 6: (a) SEM image, (b) EDX spectrum, and (c) TEM image of $\mathrm{V}_{0.9} \mathrm{Mo}_{0.05} \mathrm{~W}_{0.05}$ ONTs.

TABLE 1: The $d$ values of different samples obtained by XRD.

\begin{tabular}{|c|c|c|c|c|c|}
\hline Samples & $d_{\mathrm{XRD}}(\mathrm{nm})$ & Samples & $d_{\mathrm{XRD}}(\mathrm{nm})$ & Samples & $d_{\mathrm{XRD}}(\mathrm{nm})$ \\
\hline VONTs [9] & 3.35 & VONTs [9] & 3.35 & VONTs [9] & 3.35 \\
\hline $\mathrm{V}_{0.95} \mathrm{~W}_{0.05} \mathrm{ONTs}$ & 3.50 & $\mathrm{~V}_{0.9} \mathrm{Mo}_{0.1} \mathrm{ONTs}$ & 4.11 & $\mathrm{~V}_{0.9} \mathrm{Mo}_{0.05} \mathrm{~W}_{0.05} \mathrm{ONTs}$ & 3.84 \\
\hline $\mathrm{V}_{0.9} \mathrm{~W}_{0.1} \mathrm{ONTs}$ & 3.86 & $\mathrm{~V}_{0.8} \mathrm{Mo}_{0.2} \mathrm{ONTs}$ & 4.06 & $\mathrm{~V}_{0.8} \mathrm{Mo}_{0.1} \mathrm{~W}_{0.1}$ ONTs & 3.91 \\
\hline \multirow[t]{2}{*}{$\mathrm{V}_{0.8} \mathrm{~W}_{0.2} \mathrm{ONTs}$} & 3.94 & & & $\mathrm{~V}_{0.6} \mathrm{Mo}_{0.2} \mathrm{~W}_{0.2} \mathrm{ONTs}$ & 4.98 \\
\hline & & & & $\mathrm{V}_{0.5} \mathrm{Mo}_{0.25} \mathrm{~W}_{0.25} \mathrm{ONTs}$ & 4.77 \\
\hline
\end{tabular}

TABLE 2: The $d$ values of different samples obtained by XRD and TEM.

\begin{tabular}{lcc}
\hline Samples & $d_{\text {XRD }}(\mathrm{nm})$ & $d_{\text {TEM }}(\mathrm{nm})$ \\
\hline $\mathrm{V}_{0.95} \mathrm{~W}_{0.05}$ ONTs & 3.5 & 2.4 \\
$\mathrm{~V}_{0.9} \mathrm{Mo}_{0.05} \mathrm{~W}_{0.05}$ ONTs & 3.8 & 2.6 \\
\hline
\end{tabular}

relatively similar to that of $\mathrm{V}_{0.9} \mathrm{Mo}_{0.1} \mathrm{~W}_{0.1} \mathrm{ONTs}$. These results indicate that different elements doped into VONTs with the same doping level have nearly the same interlayer distances.

Figures 5(a) and 6(a) show SEM images of $\mathrm{V}_{0.95} \mathrm{~W}_{0.05} \mathrm{ONTs}$ and $\mathrm{V}_{0.9} \mathrm{Mo}_{0.05} \mathrm{~W}_{0.05} \mathrm{ONTs}$, respectively.
The SEM images indicate that the samples have tubular morphology and nanometric size. The chemical analyses of synthesized samples obtained by EDX are presented in Figures 5(b) and 6(b). The EDX spectra confirm the presence of vanadium, molybdenum, and tungsten in vanadium oxide nanotubes. Figures 5(c) and 6(c) show TEM images of $\mathrm{V}_{0.95} \mathrm{~W}_{0.05} \mathrm{ONTs}$ and $\mathrm{V}_{0.9} \mathrm{Mo}_{0.05} \mathrm{~W}_{0.05} \mathrm{ONTs}$, respectively. These images indicate that the morphology of both samples is tubular and multiwall. The average measured interlayer distances were 2.4 and $2.6 \mathrm{~nm}$, respectively. The interlayer distances measured using the TEM images, $d_{\text {TEM }}$, and $d_{\text {XRD }}$ values of $\mathrm{V}_{0.95} \mathrm{~W}_{0.05}$ ONTs and $\mathrm{V}_{0.9} \mathrm{Mo}_{0.05} \mathrm{~W}_{0.05} \mathrm{ONTs}$ samples are listed in Table 2. Always $d$ values obtained 
from XRD patterns are larger than those of measured from the TEM images [9]. This deviation of $d$ value might be due to a partial rearrangement of the flexible, paraffin-like arrangement of template molecules between the layers under the influence of the electron beam [9].

\section{Conclusion}

According to our results, doping of Mo, W and Mo and $\mathrm{W}$, into VONTs was successfully prepared by using sol-gel method followed by hydrothermal method, and the synthesized samples had tubular and multiwall morphology with open ends. The results showed that the interlayer distances increased with the increase of doping level of the elements into VONTs. Interlayer distances obtained from the XRD patterns, $d_{\mathrm{XRD}}$ are larger than those obtained from the TEM images, $d_{\mathrm{TEM}}$.

\section{Acknowledgments}

The authors are very grateful to A. Noorallahi and L. Anbir from refinery of Ghods city for their help in thermal treatment of the samples.

\section{References}

[1] W. Chen, L. Mai, J. Peng, Q. Xu, and Q. Zhu, "Raman spectroscopic study of vanadium oxide nanotubes," Journal of Solid State Chemistry, vol. 177, no. 1, pp. 377-379, 2004.

[2] W. Chen, L. Mai, Y. Qi, and Y. Dai, "One-dimensional nanomaterials of vanadium and molybdenum oxides," Journal of Physics and Chemistry of Solids, vol. 67, no. 5-6, pp. 896-902, 2006.

[3] C. J. Cui, G. M. Wu, H. Y. Yang et al., "A new highperformance cathode material for rechargeable lithium-ion batteries: polypyrrole/vanadium oxide nanotubes," Electrochimica Acta, vol. 55, no. 28, pp. 8870-8875, 2010.

[4] C. J. Cui, G. M. Wu, J. Shen et al., "Synthesis and electrochemical performance of lithium vanadium oxide nanotubes as cathodes for rechargeable lithium-ion batteries. , -," Electrochimica Acta, vol. 55, pp. 2536-2541, 2010.

[5] J. Emmerich, M. Dillen, C. E. A. Kirschhock, and J. A. Martens, "Fine-tuning of vanadium oxide nanotubes," Studies in Surface Science and Catalysis, vol. 175, pp. 249-252, 2010.

[6] A. V. Grigorieva, A. B. Tarasov, E. A. Goodilin et al., "Sensor properties of vanadium oxide nanotubes," Mendeleev Communications, vol. 18, no. 1, pp. 6-7, 2008.

[7] M. Roppolo, C. B. Jacobs, S. Upreti, N. A. Chernova, and M. S. Whittingham, "Synthesis and characterization of layered and scrolled amine-templated vanadium oxides," Journal of Materials Science, vol. 43, no. 14, pp. 4742-4748, 2008.

[8] F. Li, X. Wang, C. Shao, R. Tan, and Y. Liu, "W doped vanadium oxide nanotubes: synthesis and characterization," Materials Letters, vol. 61, no. 6, pp. 1328-1332, 2007.

[9] L. Q. Mai, W. Chen, Q. Xu, J. F. Peng, and Q. Y. Zhu, "Mo doped vanadium oxide nanotubes: microstructure and electrochemistry," Chemical Physics Letters, vol. 382, no. 3-4, pp. 307-312, 2003.

[10] L. F. Jiao, H. T. Yuan, Y. C. Si, Y. J. Wang, and Y. M. Wang, "Synthesis of $\mathrm{Cu} 0.1$-doped vanadium oxide nanotubes and their application as cathode materials for rechargeable magnesium batteries," Electrochemistry Communications, vol. 8, no. 6, pp. 1041-1044, 2006.

[11] W. Chen, J. Peng, L. Mai, Q. Zhu, and Q. Xu, "Synthesis of vanadium oxide nanotubes from $\mathrm{V}_{2} \mathrm{O}_{5}$ sols," Materials Letters, vol. 58, no. 17-18, pp. 2275-2278, 2004.

[12] L. Mai, W. Chen, Q. Xu, Q. Zhu, C. Han, and J. Peng, "Costsaving synthesis of vanadium oxide nanotubes," Solid State Communications, vol. 126, no. 10, pp. 541-543, 2003.

[13] F. Sediri, F. Touati, and N. Gharbi, "A one-step hydrothermal way for the synthesis of vanadium oxide nanotubes containing the phenylpropylamine as template obtained via non-alkoxide route," Materials Letters, vol. 61, no. 8-9, pp. 1946-1950, 2007. 

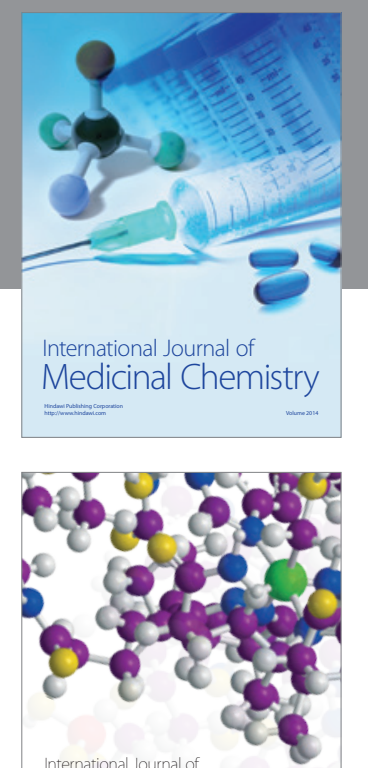

\section{Carbohydrate} Chemistry

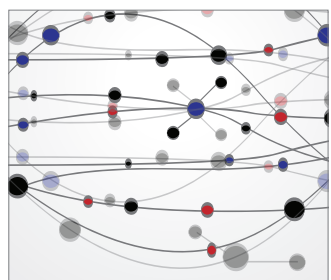

The Scientific World Journal
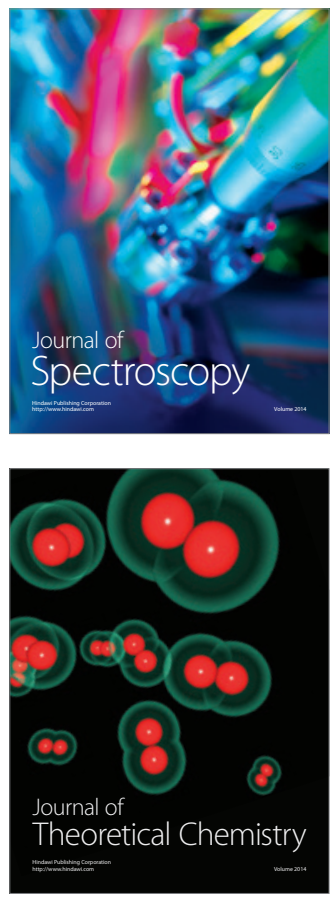
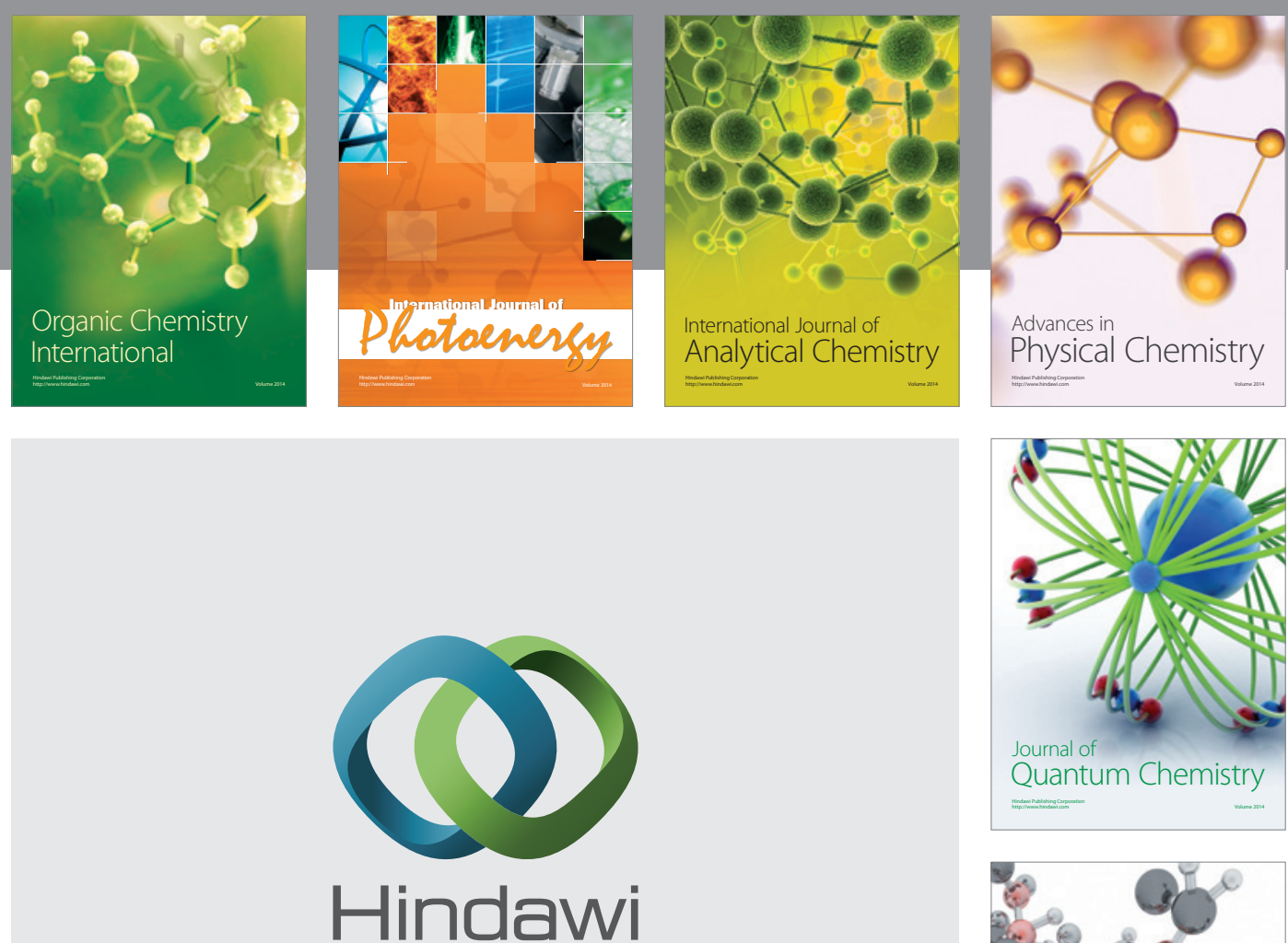

Submit your manuscripts at

http://www.hindawi.com

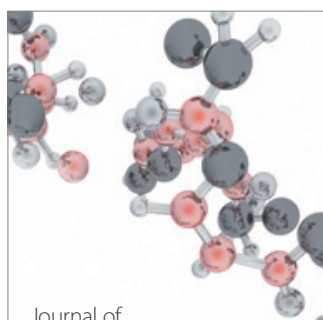

Analytical Methods

in Chemistry

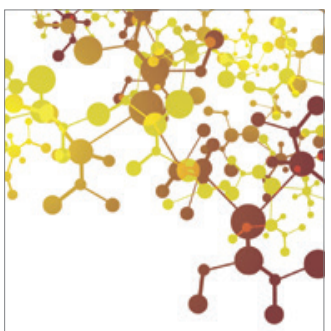

Journal of

Applied Chemistry

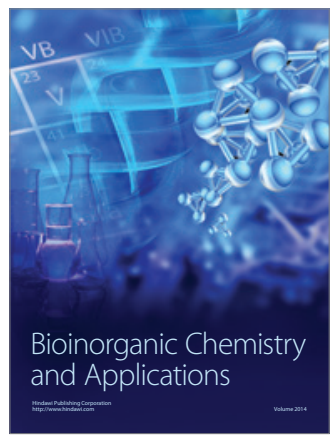

Inorganic Chemistry
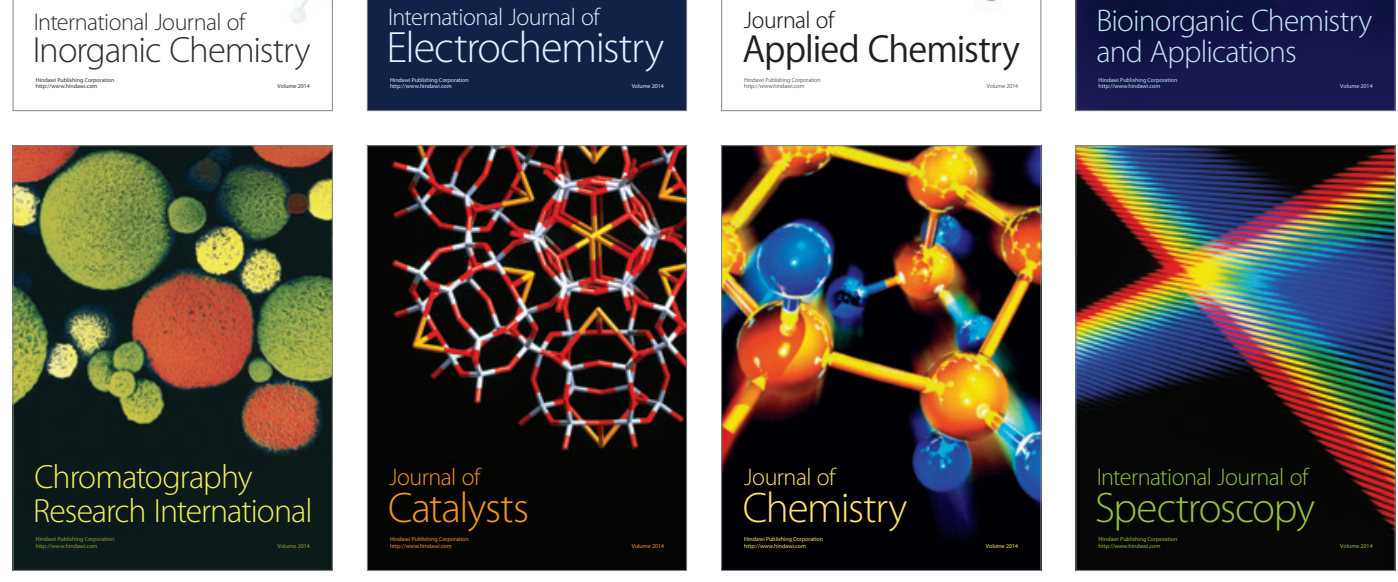